\title{
Generation of Tunable Microwave Frequency Comb Utilizing a Semiconductor Laser Subject to Optical Injection from an SFP Module Modulated by an Arbitrary Periodic Signal
}

\author{
Qingchun Zhao (D), Maohai Zhai, Wenbo Shi, and Bin Wu \\ School of Computer and Communication Engineering, Northeastern University at Qinhuangdao, Qinhuangdao, \\ Hebei 066004, China \\ Correspondence should be addressed to Qingchun Zhao; zhaoqingchun2000@163.com
}

Received 14 April 2020; Accepted 15 May 2020; Published 8 June 2020

Guest Editor: Shao-Bo He

Copyright ( ) 2020 Qingchun Zhao et al. This is an open access article distributed under the Creative Commons Attribution License, which permits unrestricted use, distribution, and reproduction in any medium, provided the original work is properly cited.

\begin{abstract}
In this paper, a microwave frequency comb (MFC) is generated from a semiconductor laser subject to optical injection from a commercial small form-factor pluggable (SFP) optical module which is modulated by an arbitrary periodic signal. A sinusoidal signal or square wave signal is employed as the arbitrary periodic signal instead of the electric pulse signal adopted in the former references. When the frequency of the modulated signal is $1 \mathrm{GHz}$, the MFC with a maximal bandwidth of $15 \mathrm{GHz}$ can be obtained. In addition, taking a sinusoidal signal as an example, the influence of the injection optical power to the slave laser and the modulation frequency of the optical module on the generation of the MFC is analyzed in detail. Finally, the results of MFC generated with a square wave signal injection are presented. The experimental results of this paper provide an important reference for the practical applications of MFC.
\end{abstract}

\section{Introduction}

With the external disturbances such as external optical injection, optical feedback, or opto-electric feedback, a semiconductor laser can produce some nonlinear dynamic phenomena, including period one oscillation $[1,2]$, perioddoubling oscillation, quasiperiod oscillation, and chaos [3-8]. In addition, a semiconductor laser can also generate microwave frequency comb (MFC) under the external optical injection. MFC is widely used in frequency measurement, radar detection, frequency conversion, radio-overfiber (RoF) transmission system, and other fields due to the advantages of multiple continuous microwave signals, flexible and adjustable comb line spacing, wide frequency range, and high comb spacing precision $[2,9-11]$. Therefore, generation of stable and high-quality MFC has become a research hotspot.

Some methods of generating MFC have been reported up to now. The MFC signal with a bandwidth of $3 \mathrm{GHz}$ was generated by using the subharmonic frequency locked state generated by the opto-electric feedback of semiconductor laser [12]. But the comb spacing of the frequency comb was not strictly equal. Moreover, the noise of noncomb component was obvious. By injecting the regular optical pulse generated by opto-electric feedback of semiconductor laser into the other semiconductor lasers, the MFC signal was generated with an amplitude of $\pm 5 \mathrm{~dB}$ and bandwidth of $20 \mathrm{GHz}[13,14]$. However, the signal was unstable, and the comb line spacing was small. By adopting the nonlinear effect of STM tunnel junction, the generation of the MFC signal with up to 200 harmonics was realized. The central frequency of the highest harmonic is $14.85 \mathrm{GHz}$. Yet there are some disadvantages such as difficult to tune the comb spacing and large phase noise [15-17]. In addition, the numerical simulations and experiments have been presented for the generation of tunable MFC signal by semiconductor laser subject to optical injection, in which the master laser is modulated by a periodic electric pulse signal [18-20]. 
In this paper, a tunable MFC signal is obtained by using an external optical injection semiconductor laser. The master laser is a commercial SFP optical module modulated by a periodic electronic signal not just a periodic electric pulse signal used in [20]. The modulation signal in this experiment is the sinusoidal signal produced by a RF signal generator or the square wave signal produced by a bit-error-rate tester (BERT). As the master laser, in order to reduce the cost and the complexity of the setup, we use a commercial optical module which is cheap, simple, and easy to be modulated.

\section{Experimental Setup}

The experimental setup of the tunable MFC produced by a semiconductor laser subject to the optical injection from a SFP optical module modulated by a sinusoidal signal is presented in Figure 1. The master laser is a $2.5 \mathrm{~Gb} / \mathrm{s}$ small form-factor pluggable (SFP, Finisar) optical module, yet the slave laser is a distributed feedback (DFB) semiconductor laser without a build-in optical isolator. The optical power and central wavelength of the slave laser are controlled by the current source circuit and the temperature control circuit, respectively. The SFP module generates an optical signal that changes with the modulation of a sinusoidal signal from a RF signal generator (SG, IFR 2042, 5.4 GHz). After being amplified by erbium-doped fiber amplifier (EDFA), the light enters the variable optical attenuator (VOA) to adjust the injected optical power. A polarization controller (PC) is adopted to adjust the polarization state. Then, the light enters the slave laser through the optical circulator (OC) to generate a new optical signal utilizing nonlinear interaction. A 50:50 optical coupler is adopted to divide light into two beams. One beam enters a photodetector (PD, Picometrix, PT-40D/AC) with a bandwidth of $40 \mathrm{GHz}$ to convert the optical signal into electrical signal. The output electrical signal is gathered by the electrical spectrum analyzer (ESA, Rohde \& Schwarz, FSEK20, $40 \mathrm{GHz}$ ). The other beam enters the optical spectrum analyzer (OSA, Anritsu, MS9710C) to analyze the optical spectrum.

The frequency range of the sinusoidal signal generated from the signal generator is changed from $0.5 \mathrm{GHz}$ to $5.4 \mathrm{GHz}$ in this experiment. The SFP is an optical module that meets the dense wavelength division multiplexing (DWDM) wavelength requirements. The actual output wavelength is $1549.76 \mathrm{~nm}$. In order to adjust the injected optical power for a large range, the EDFA is necessary. The output power of the SFP is small, but the power can be amplified to $11.3 \mathrm{dBm}$ by EDFA working under the automatic power control (APC). The VOA is employed to adjust the optical power injecting into the slave laser with an initial attenuation coefficient of $11 \mathrm{~dB}$. The bias voltage of the photodetector with a transimpedance amplifier is $+4 \mathrm{~V}$, yet the voltage for the transimpedance amplifier is $+3.3 \mathrm{~V}$. For this experiment, the optimal MFC signal is obtained by adjusting the frequency of the sinusoidal signal from the signal generator, the $\mathrm{PC}$, and the VOA.

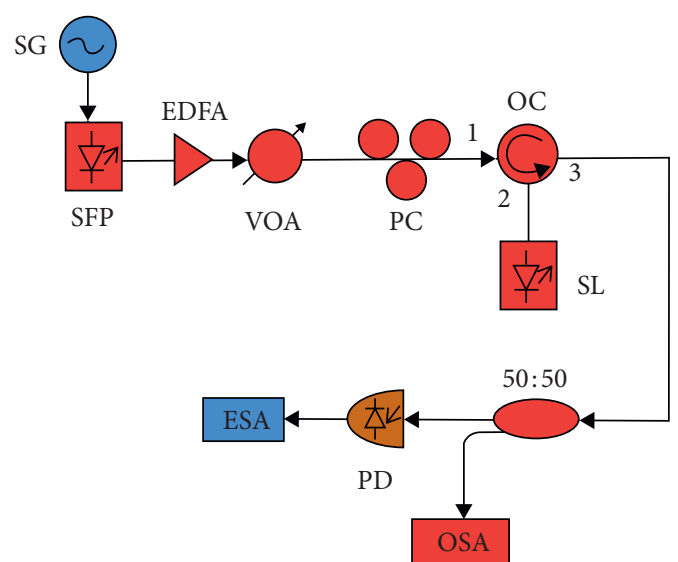

FIGURE 1: Experimental setup. SFP: small form-factor pluggable; EDFA: erbium-doped fiber amplifier; VOA: variable optical attenuator; PC: polarization controller; OC: optical circulator; SL: slave laser; PD: photodetector; ESA: electrical spectrum analyzer; OSA: optical spectrum analyzer; SG: signal generator.

\section{Effect of the Injection Optical Power on MFC}

The initial frequency of the signal generator is $1 \mathrm{GHz}$ with an amplitude of $+0.7 \mathrm{~V}$. The attenuation coefficient of the VOA is between $11 \mathrm{~dB}$ and $25 \mathrm{~dB}$ with an attenuation step of $1 \mathrm{~dB}$. When the attenuator coefficient is $11 \mathrm{~dB}$, the obtained MFC is shown in Figure 2(a). The comb line spacing of the MFC is equal to the frequency of the modulation signal, which is $1 \mathrm{GHz}$. The bandwidth of MFC can be determined by the electrical spectrum analyzer as follows. Firstly, the function of electrical spectrum analyzer to find the peak value is used to directly locate the component with the highest power for the frequency comb. The frequency of the component is $3 \mathrm{GHz}$, and the power is $-27.95 \mathrm{dBm}$, which is the third comb line of the MFC. In this paper, the bandwidth for the MFC is defined as $10 \mathrm{~dB}$ [20], that is, the maximum power value minus $10 \mathrm{dBm}$. Then, the MFC component within this range is the bandwidth. Accordingly, an isoline with a power of $-27.95 \mathrm{dBm}$ is drawn to determine the upper bound. Secondly, an isoline with the power of $-37.95 \mathrm{dBm}$ is drawn to determine the lower bound. It can be seen from Figure 2(a) that there are 15 comb line components between the two power contour lines. Therefore, the bandwidth of the MFC is $15 \mathrm{GHz}$. The peak power difference for each comb line is small. Hence, the MFC signal quality is good. Although there is a frequency component greater than $15 \mathrm{GHz}$, the signal power is lower than $-37.95 \mathrm{dBm}$, which is not considered in the bandwidth.

The MFC signal is shown in Figure 2(b) when the attenuation coefficient of the VOA is set to $20 \mathrm{~dB}$. The frequency of the modulation signal is $1 \mathrm{GHz}$; hence, the frequency of the generated MFC signal is an integral multiple of $1 \mathrm{GHz}$, that is, from $1 \mathrm{GHz}$ and $2 \mathrm{GHz}$ to $11 \mathrm{GHz}$. Except these frequencies, noise appears. It can be seen that there is a lot of noise at the bottom of some frequency comb lines. The frequency comb with the high frequency part is almost submerged by noise. The signal quality is not as good, as shown in Figure 2(a), but the MFC with a wide spectrum 


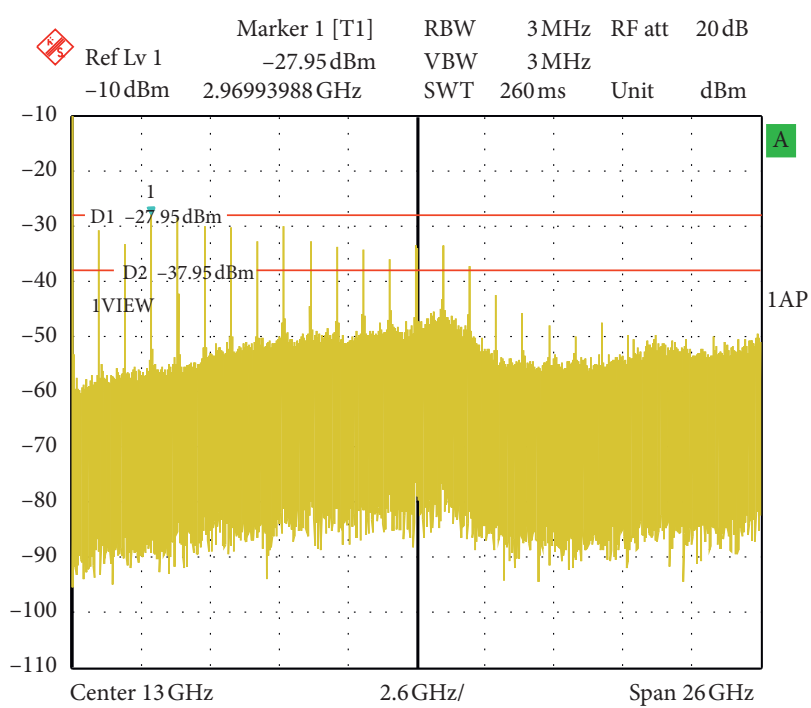

(a)

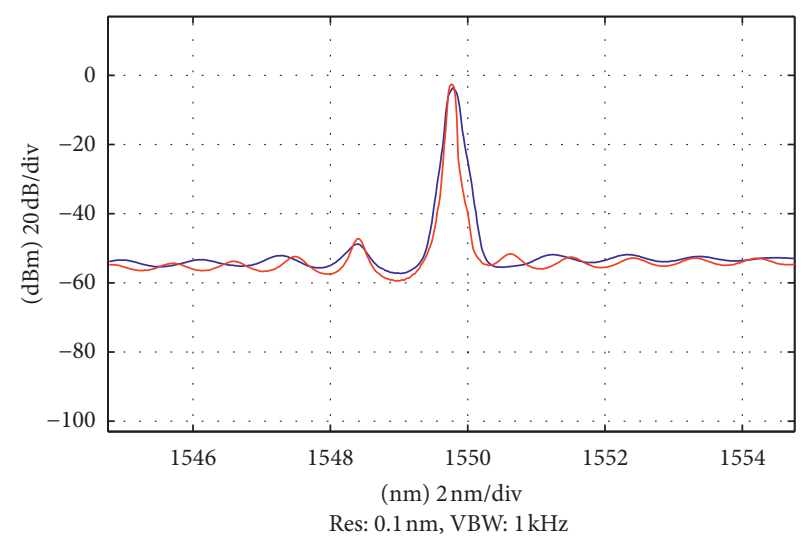

$-\mathrm{A}$

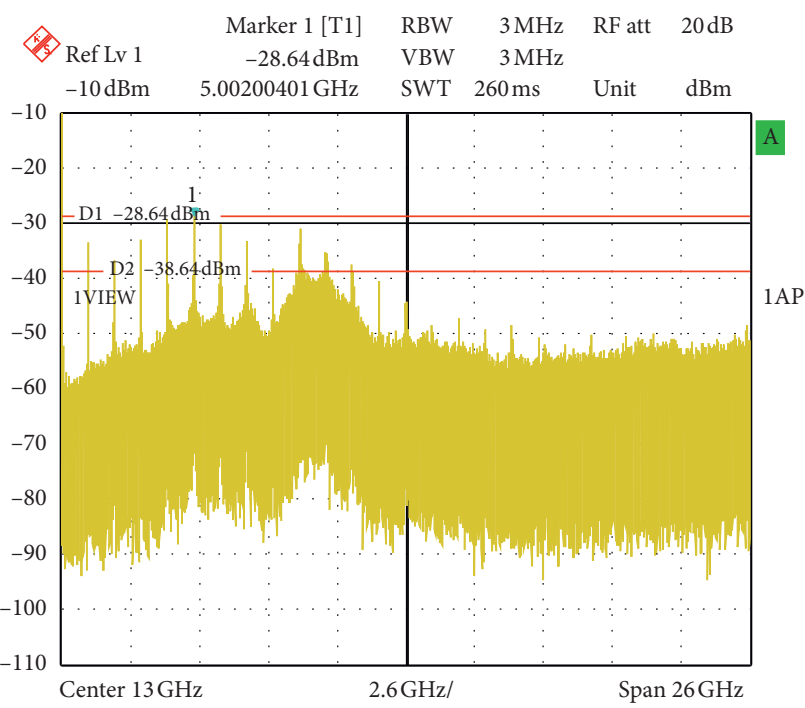

(b)

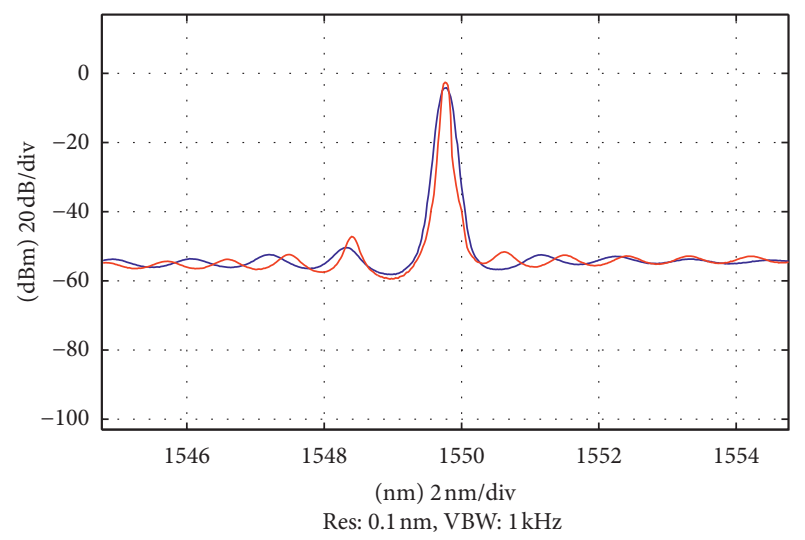

(d)

FIGURE 2: Electrical spectrum of the MFC when the modulation signal frequency is $1 \mathrm{GHz}$ and the attenuator coefficient of VOA is $11 \mathrm{~dB}$ (a) and $20 \mathrm{~dB}(\mathrm{~b})$. The optical spectrum when the attenuator coefficient of VOA is $11 \mathrm{~dB}$ (c) and $20 \mathrm{~dB}$ (d) For (c) and (d), curve A denotes the optical spectrum of the optical signal generated from SL with the injection from SFP module. Curve B denotes the optical spectrum of the SFP module.

can still be obtained. According to the above method of bandwidth determination, it can be concluded that the bandwidth of the MFC is $11 \mathrm{GHz}$ for this case.

For this experiment, we also measured the optical spectrum of the optical signal generated from SL after optical injection. Figure 2(c) is the optical spectrum when the attenuation coefficient of the VOA is $11 \mathrm{~dB}$. Curve $\mathrm{A}$ is the optical spectra of the optical signal generated from SL after optical injection, while curve B denotes the optical spectra of the SFP module. It can be seen that the central wavelength of the optical signal generated from SL after optical is equal to that of the SFP module. That is to say, when the central wavelength of the master laser and the slave laser is equal, the MFC can be obtained. Figure 2(d) is the optical spectrum of the VOA when the attenuation coefficient is $20 \mathrm{~dB}$. It can be seen that the optical spectrum hardly changes with the increasing attenuation coefficient.
The injection optical power from the SFP module decreases gradually by adjusting the attenuation of VOA. The bandwidth of the MFC changes with the attenuation coefficient, as shown in Figure 3. When the attenuation coefficient is from $11 \mathrm{~dB}$ to $13 \mathrm{~dB}$ or from $24 \mathrm{~dB}$ to $25 \mathrm{~dB}$, the bandwidth of the MFC does not change. When the attenuation coefficient is from $14 \mathrm{~dB}$ to $24 \mathrm{~dB}$, the MFC bandwidth presents a general decreasing trend with the increasing attenuation coefficient. Therefore, the bandwidth of the MFC generated in this experiment generally decreases with the decrease in the output power of the SFP module, but the overall fluctuation is not big.

\section{Effect of Modulation Frequency on MFC}

During this section, the attenuation coefficient of VOA is maintained at $11 \mathrm{~dB}$. Figure $4(\mathrm{a})$ shows the generated MFC 


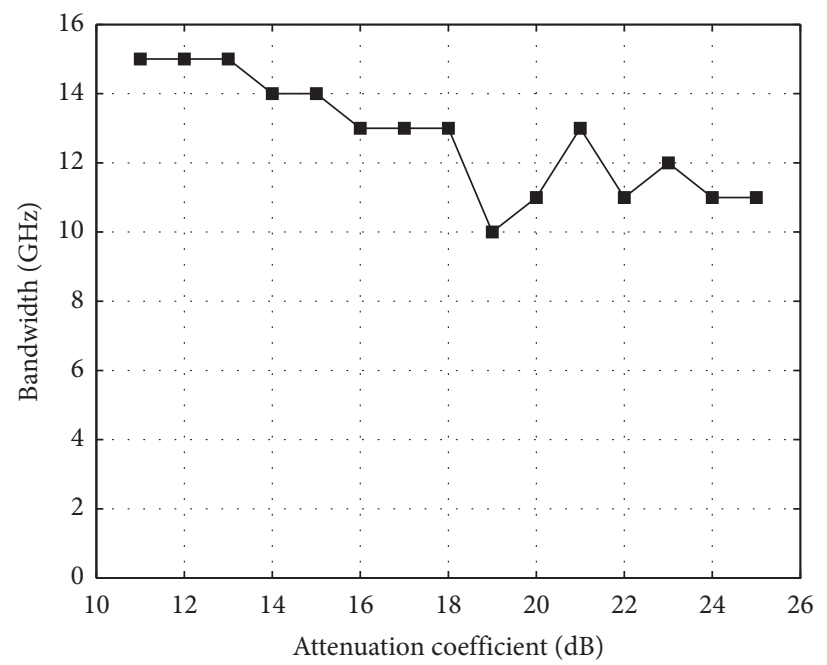

Figure 3: Influence of the output optical power of the SFP optical module on the MFC. The $X$-axis is represented by the attenuation coefficient of the VOA.

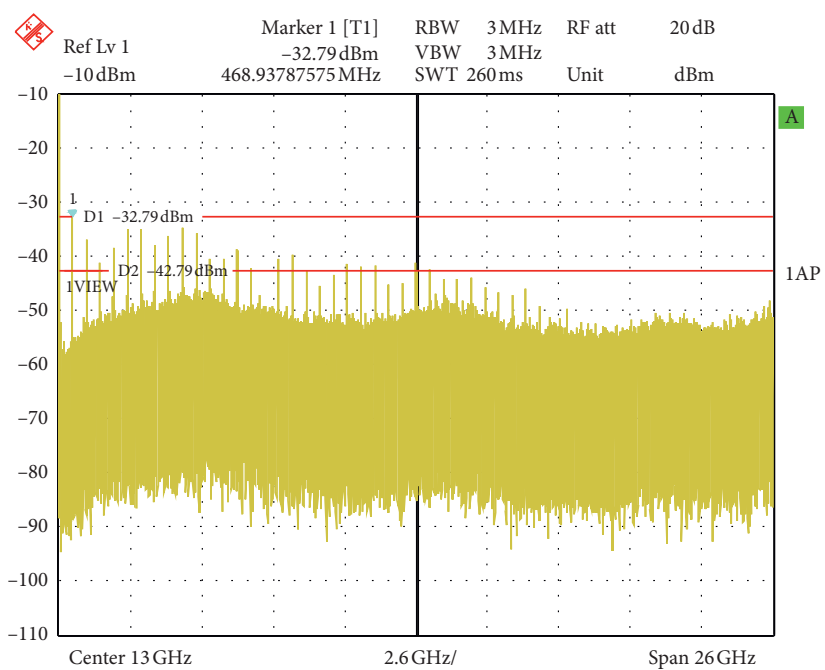

(a)

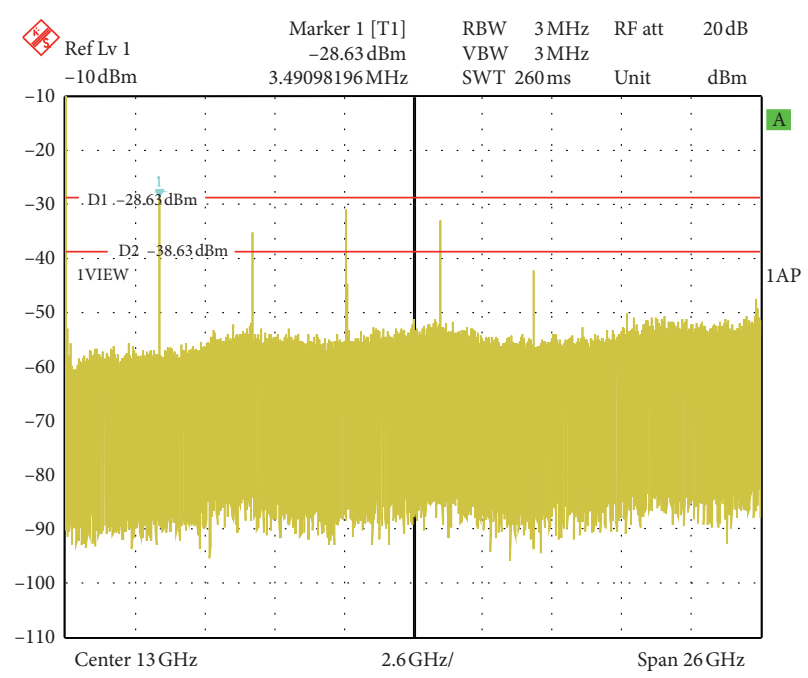

(b)

FIGURE 4: Generated MFC when the modulation frequency is $0.5 \mathrm{GHz}$ (a) and $3.5 \mathrm{GHz}$ (b).

when the modulation frequency from the signal generator is $0.5 \mathrm{GHz}$. It can be seen that the comb line spacing of the MFC is equal to the frequency of the modulation signal, i.e., $0.5 \mathrm{GHz}$. Compared with the result of $1 \mathrm{GHz}$ modulation frequency in Figure 2(a), when the modulation frequency is $0.5 \mathrm{GHz}$, the power of the low-frequency base part increases, so the quality of the obtained MFC decreases. The bandwidth for the MFC shown in Figure 4(a) is $7 \mathrm{GHz}$. The obtained MFC is shown in Figure 4(b) when the output frequency of the signal generator is $3.5 \mathrm{GHz}$. The comb line spacing is $3.5 \mathrm{GHz}$, which equals to the sinusoidal signal modulation frequency. However, the frequency comb of the high frequency part disappears. As shown in Figure 4(b), the MFC whose overall base is flat is better than the result presented in Figure 4(a). The bandwidth for the MFC shown in Figure 4(b) is $14 \mathrm{GHz}$.

The relationship between the bandwidth of the MFC and the modulation frequency is displayed in Figure 5. With the increasing modulation frequency, the bandwidth of MFC is generally increasing. When the modulation frequency is between $1 \mathrm{GHz}$ and $2 \mathrm{GHz}$, the bandwidth decreases slightly. The quality of the MFC is not very good. Generally speaking, when the range of the modulation frequency is from $2.5 \mathrm{GHz}$ to $5 \mathrm{GHz}$, the bandwidth begins to increase. Moreover, not only the noise is relatively small and the comb line is clean but also the power distribution of each comb line is relatively uniform.

\section{MFC Generated with Square Wave Signal Modulation}

In order to illustrate that a MFC signal can be generated from a semiconductor laser subject to optical injection from a SFP module modulated by arbitrary periodic signal, a square wave signal is employed as the modulated signal. 


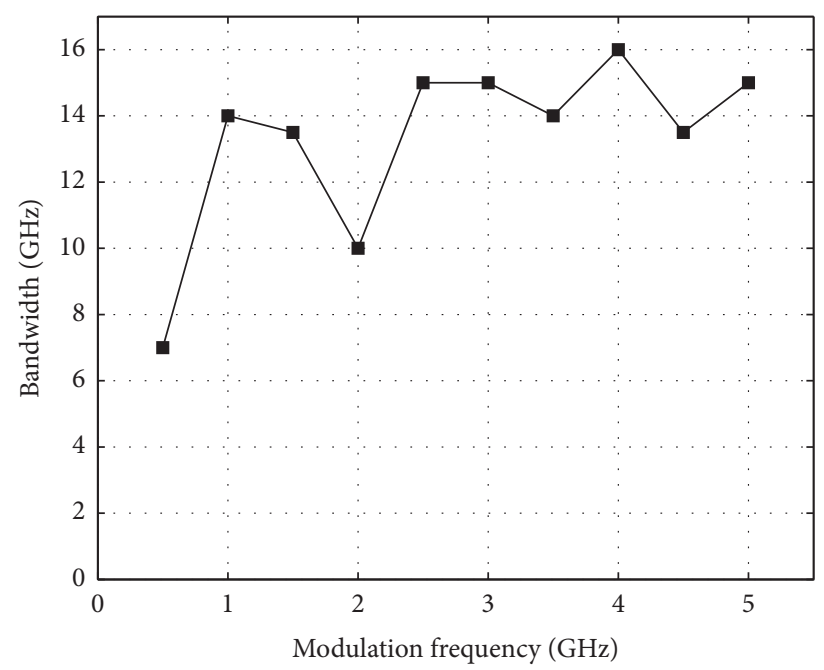

Figure 5: Effect of modulation frequency on MFC.

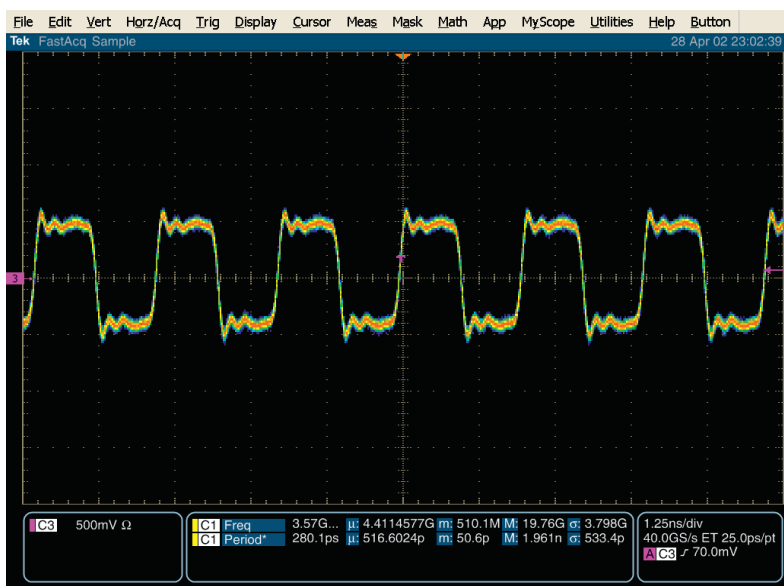

(a)

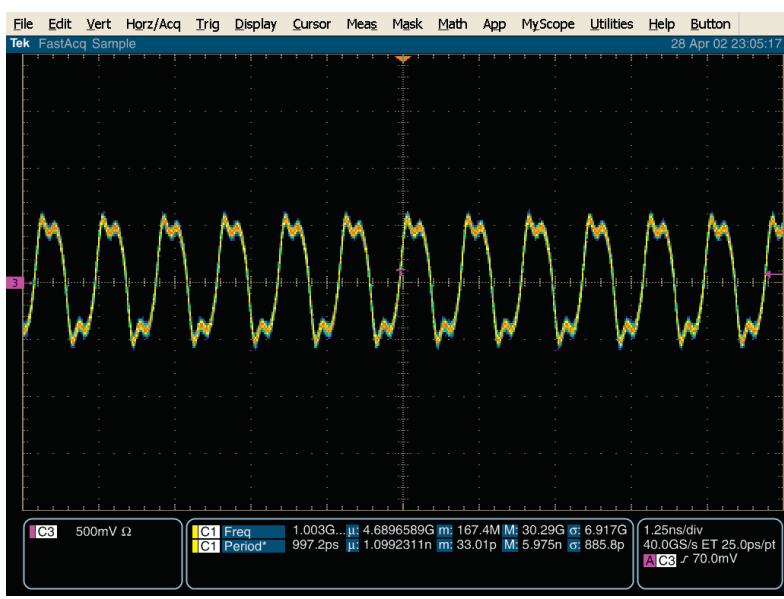

(c)

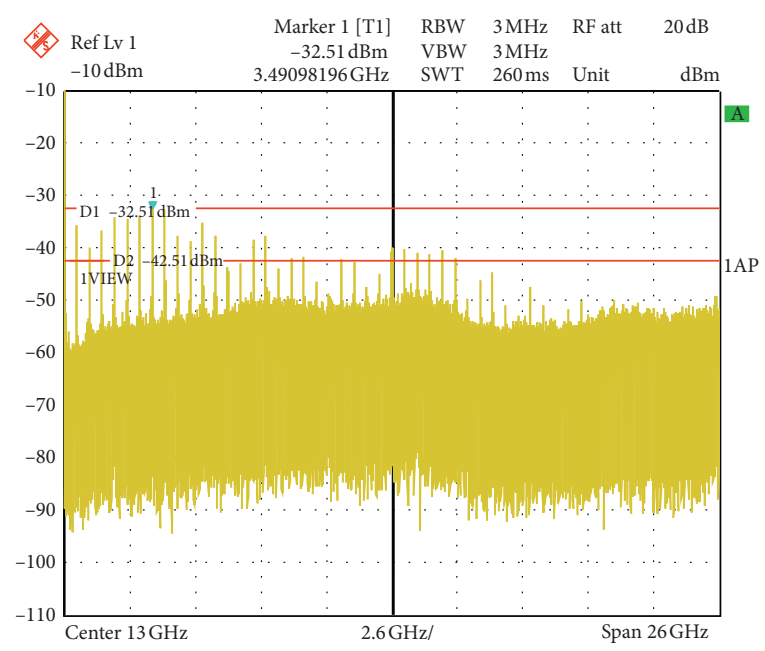

(b)

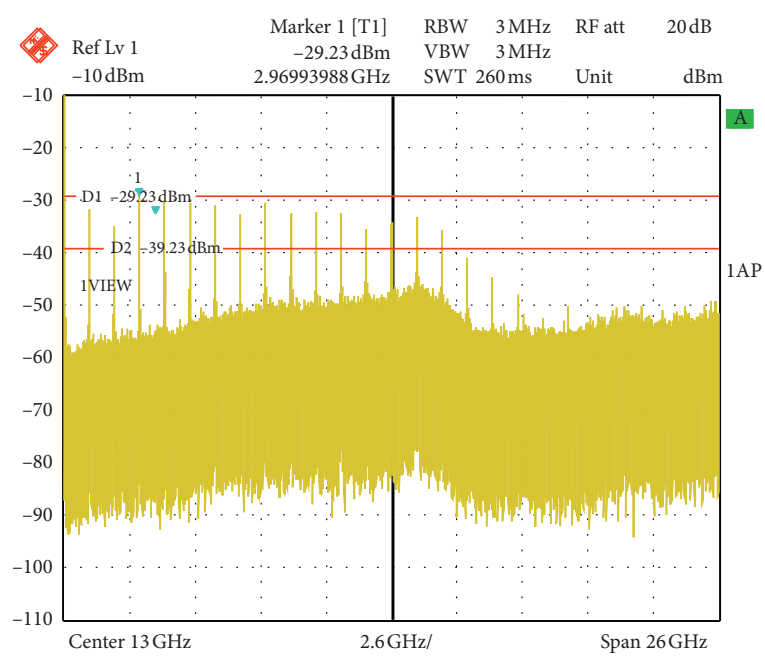

(d)

FigURE 6: Results of the MFC generated by the square wave signal modulation. (a) and (c) are the $0.5 \mathrm{GHz}$ and $1 \mathrm{GHz}$ square wave signals, respectively. (b) and (d) are the generated MFC with the modulation of $0.5 \mathrm{GHz}$ and $1 \mathrm{GHz}$ square wave signals, respectively. 
During this experiment, a bit-error-rate tester (BERT, Anritsu, MP1632A, $3.2 \mathrm{~Gb} / \mathrm{s}$ ) is utilized to generate a square wave signal. By the way, a bit-error-rate tester is generally arranged to generate the pseudorandom bit sequence. The specific setting is to utilize the pattern setting function in the pattern editor menu of the BERT. The data output from the pulse pattern generator (PPG) of the BERT is the square wave signal when the running mode is selected as programmable pattern (PRGM) and set the pattern type as periodic 0 and 1 . The time-domain waveform is displayed on a real-time digital oscilloscope (OSC, Tektronix, TDS7404B, $4 \mathrm{GHz}, 20 \mathrm{GS} / \mathrm{s}$ ).

The square wave signal generated by the BERT is employed to modulate the SFP module. Figure 6(a) presents the square wave signal with the output frequency of $0.5 \mathrm{GHz}$. Figure 6(b) displays the corresponding MFC signal generated by slave laser with the optical injection from SFP module with the modulation of the square wave signal. For this case, the bandwidth of MFC signal is $6 \mathrm{GHz}$. Comparing the MFC obtained this time with that obtained from the sinusoidal signal with the modulation frequency of $0.5 \mathrm{GHz}$, the bandwidth is $6 \mathrm{GHz}$ and $7 \mathrm{GHz}$, respectively. Not only the difference of the bandwidth is not big but also the main characteristics of the base noise distribution, comb line, and comb line power distribution of the MFC are similar.

When the frequency of the square wave signal is $1 \mathrm{GHz}$, the results of the oscilloscope and the electronic spectra are shown in Figures 6(c) and 6(d), respectively. The comb line spacing of the MFC signal increases from $0.5 \mathrm{GHz}$ to $1 \mathrm{GHz}$ when the square wave signal is $1 \mathrm{GHz}$. The number of the comb lines decreases, and the power of comb lines component within the bandwidth increases. Hence, the MFC signal quality generally improves. When the frequency of modulation signal is $1 \mathrm{GHz}$, the bandwidth of the MFC signal obtained with the square wave signal and the sinusoidal signal is $15 \mathrm{GHz}$ and $14 \mathrm{GHz}$, respectively. The results of the two MFC signals on the electronic spectrum analyzer are approximately the same. This is a strong proof of the argument put forward in this paper; that is, the MFC can be generated from a semiconductor laser with optical injection from a modulated SFP module by arbitrary periodic signal.

Other waveforms, such as triangle-wave signal or periodic pulse signal, can also be adopted as the modulated signal, which can be generated by arbitrary waveform generator (AWG) with high bandwidth. At present, we have no such high-bandwidth AWG in our laboratory. In future, we will conduct some experiments to generate MFC with the modulation signal produced by AWG.

\section{Conclusions}

In this paper, a sinusoidal signal or square wave signal is employed as a periodic signal to modulate the SFP module in order to realize the generation of the microwave frequency comb signal from the semiconductor laser with the injection from the SFP module. The proposed setup can generate stable microwave frequency comb signal in the range of $6 \mathrm{GHz}-16 \mathrm{GHz}$. The results of this paper provide a useful reference for the practical application of the microwave frequency comb signal.

\section{Data Availability}

The data used to support the findings of this study are available from the corresponding author upon request.

\section{Conflicts of Interest}

The authors declare that there are no conflicts of interest regarding the publication of this paper.

\section{Acknowledgments}

This work was supported by the Natural Science Foundation of China (NSFC) (61602099 and U1708262).

\section{References}

[1] S. Ji, Y. Hong, P. S. Spencer, J. Benedikt, and I. Davies, "Broad tunable photonic microwave generation based on period-one dynamics of optical injection vertical-cavity surface-emitting lasers," Optics Express, vol. 25, no. 17, pp. 19863-19871, 2017.

[2] C. Cui and S.-C. Chan, "Performance analysis on using period-one oscillation of optically injected semiconductor lasers for radio-over-fiber uplinks," IEEE Journal of Quantum Electronics, vol. 48, no. 4, pp. 490-499, 2012.

[3] T. B. Simpson, J. M. Liu, K. F. Huang, and K. Tai, "Nonlinear dynamics induced by external optical injection in semiconductor lasers," Quantum and Semiclassical Optics: Journal of the European Optical Society Part B, vol. 9, no. 5, pp. 765-784, 1997.

[4] F.-Y. Lin, S.-Y. Tu, C.-C. Huang, and S.-M. Chang, "Nonlinear dynamics of semiconductor lasers under repetitive optical pulse injection," IEEE Journal of Selected Topics in Quantum Electronics, vol. 15, no. 3, pp. 604-611, 2009.

[5] Y.-S. Juan and F.-Y. Lin, "Photonic generation of broadly tunable microwave signals utilizing a dual-beam optically injected semiconductor laser," IEEE Photonics Journal, vol. 3, no. 4, pp. 644-650, 2011.

[6] J.-W. Wu, Q. Qiu, X.-P. Zhang, and Y. H. Won, "Simultaneous generation of microwave, millimeter-wave, and terahertz photonic signal based on two-color semiconductor laser subject to single-beam optical injection," IEEE Journal of Selected Topics in Quantum Electronics, vol. 23, no. 4, pp. 1-8, 2017.

[7] J.-P. Zhuang, X.-Z. Li, S.-S. Li, and S.-C. Chan, "Frequencymodulated microwave generation with feedback stabilization using an optically injected semiconductor laser," Optics Letters, vol. 41, no. 24, pp. 5764-5767, 2016.

[8] K.-H. Lo, S.-K. Hwang, and S. Donati, "Optical feedback stabilization of photonic microwave generation using periodone nonlinear dynamics of semiconductor lasers," Optics Express, vol. 22, no. 15, pp. 18648-18661, 2014.

[9] Y.-H. Hung and S.-K. Hwang, "Photonic microwave stabilization for period-one nonlinear dynamics of semiconductor lasers using optical modulation sideband injection locking," Optics Express, vol. 23, no. 5, pp. 6520-6532, 2015.

[10] S.-K. Hwang, C.-Y. Lin, and D.-H. Liang, "All-optical frequency conversion using nonlinear dynamics of semiconductor lasers subject to external optical injection," in 
Proceeding of the Semiconductor Lasers and Laser Dynamics III, vol. 6997, Strasbourg, France, May 2008.

[11] Y. Matsui, R. Schatz, T. Pham et al., " $55 \mathrm{GHz}$ bandwidth distributed reflector laser," Journal of Lightwave Technology, vol. 35, no. 3, pp. 397-403, 2017.

[12] S.-C. Chan, G.-Q. Xia, and J.-M. Liu, "Optical generation of a precise microwave frequency comb by harmonic frequency locking," Optics Letters, vol. 32, no. 13, pp. 1917-1919, 2007.

[13] Y.-S. Juan and F.-Y. Lin, "Microwave-frequency-comb generation utilizing a semiconductor laser subject to optical pulse injection from an optoelectronic feedback laser," Optics Letters, vol. 34, no. 11, pp. 1636-1638, 2009.

[14] Y.-S. Juan and F.-Y. Lin, "Ultra broadband microwave frequency combs generated by an optical pulse-injected semiconductor laser," Optics Express, vol. 17, no. 21, pp. 18596-18605, 2009.

[15] M. J. Hagmann, A. Efimov, A. J. Taylor, and D. A. Yarotski, "Microwave frequency-comb generation in a tunneling junction by intermode mixing of ultrafast laser pulses," Applied Physics Letters, vol. 99, no. 1, Article ID 011112, 2011.

[16] M. J. Hagmann, A. J. Taylor, and D. A. Yarotski, "Observation of 200th harmonic with fractional linewidth of 10-10 in a microwave frequency comb generated in a tunneling junction," Applied Physics Letters, vol. 101, no. 24, Article ID 241102, 2012.

[17] M. J. Hagmann, F. S. Stenger, and D. A. Yarotski, "Linewidth of the harmonics in a microwave frequency comb generated by focusing a mode-locked ultrafast laser on a tunneling junction," Journal of Applied Physics, vol. 114, no. 22, Article ID 223107, 2013.

[18] L. Fan, G.-Q. Xia, X. Tang et al., "Tunable ultra-broadband microwave frequency combs generation based on a current modulated semiconductor laser under optical injection," IEEE Access, vol. 5, pp. 17764-17771, 2017.

[19] X.-Q. Xu, L. Fan, G.-Q. Xia, and Z.-M. Wu, "Numerical investigation on ultra-broadband tunable microwave frequency comb generation using a semiconductor laser under regular pulse injection," IEEE Access, vol. 6, pp. 55284-55290, 2018.

[20] L. Fan, G.-Q. Xia, T. Deng et al., "Generation of tunable and ultra-broadband microwave frequency combs based on a semiconductor laser subject to pulse injection from a current modulated laser," IEEE Photonics Journal, vol. 10, no. 6, pp. 1-10, 2018. 